\title{
Spatial scaling of optical fluctuations during substorm-time aurora
}

\author{
B. V. Kozelov ${ }^{1,2}$ and K. Rypdal ${ }^{2}$ \\ ${ }^{1}$ Polar Geophysical Institute, Apatity, Murmansk region, 184209 Russia \\ ${ }^{2}$ Department of Physics and Technology, University of Troms $\varnothing$, 9037, Troms $\varnothing$, Norway
}

Received: 28 October 2006 - Revised: 15 March 2007 - Accepted: 20 March 2007 - Published: 8 May 2007

\begin{abstract}
A study of statistical features of auroras during substorm activity is presented, emphasizing characteristics which are commonly applied to turbulent flows. Data from all-sky television (TV) observations from the Barentsburg observatory (Svalbard) have been used. Features of the probability density function (PDF) of auroral fluctuations have been examined at different spatial scales. We find that the observed PDFs generally have a non-Gaussian, heavy-tailed shape. The generalized structure function (GSF) for the auroral luminosity fluctuations has been analyzed to determine the scaling properties of the higher (up to 6) order moments, and the evolution of the scaling indices during the actual substorm event has been determined. The scaling features obtained can be interpreted as signatures of turbulent motion of the magnetosphere-ionosphere plasma. Relations to previously obtained results of avalanche analysis of the same event, as well as possible implications for the validity of self-organized criticality models and turbulence models of the substorm activity, are discussed.
\end{abstract}

Keywords. Magnetospheric physics (Auroral phenomena) Space plasma physics (Nonlinear phenomena; Turbulence)

\section{Introduction}

During the last decade, it has become increasingly more accepted that a physical description of the magnetosphereionosphere system requires some kind of complex system approach. One class of such approaches is based on the notion of self-organized criticality (SOC), which was first proposed in a general context by Bak et al. (1988). It was introduced as a universal feature of threshold systems with a large number of degrees of freedom, in which the basic interactions are local, but where long-range interactions develop due to the

Correspondence to: B. V. Kozelov

(boris.kozelov@gmail.com) tendency for the formation of avalanches when the system approaches the critical state where it is everywhere a near instability threshold. In this state all scales interact with each other, and the dynamics of the system on different spatial scales is self-similar. This self-similarity is manifested as a power relationship between various characteristics and the temporal/spatial scale. There are many indications that the magnetospheric-ionospheric system (MIS) can exhibit characteristics typical for SOC systems (Vörös, 1991; Takalo et al., 1993; Milovanov et al., 1996; Consolini, 1997; Sharma, 1997; Uritsky and Pudovkin, 1998; Uritsky et al., 2002; Vörös et al., 2003; Kozelov et al., 2004). However, at least some of these indications have alternatively been interpreted in terms of intermittent turbulence (Borovsky and Funsten, 2003; Sorriso-Valvo et al., 2001). It should also be kept in mind that the SOC concept was also applied to describe the intermittency in the Earth's plasma sheet (Angelopoulos et al., 1999). However, the turbulence in the magnetosphereionosphere plasma also exhibits some peculiarities, which are not explained by present theoretical models (Borovsky et al., 1997; Borovsky and Funsten, 2003). Chang (2004) proposed a scenario where intermittent turbulence is formed by a dynamical topological complexity resulting from the nonlinear evolution of multi-scale coherent structures. The individual plasma processes are incorporated by this scenario in a uniform SOC-like state.

In this paper we have used the same data set which was previously analyzed in Kozelov et al. (2004). In that paper spatio-temporal techniques were employed to identify and select avalanche-like transients. Here we present a description of statistical features of aurora luminosity fluctuations during substorm activity, by focusing on characteristics which usually apply to turbulent flows: probability density function (PDF) and generalized structure function (GSF). The observations and the data set are described in Sect. 2. The shape of the PDFs of the auroral fluctuations is examined at different spatial scales in Sect. 3. Here we also illustrate

Published by Copernicus GmbH on behalf of the European Geosciences Union. 


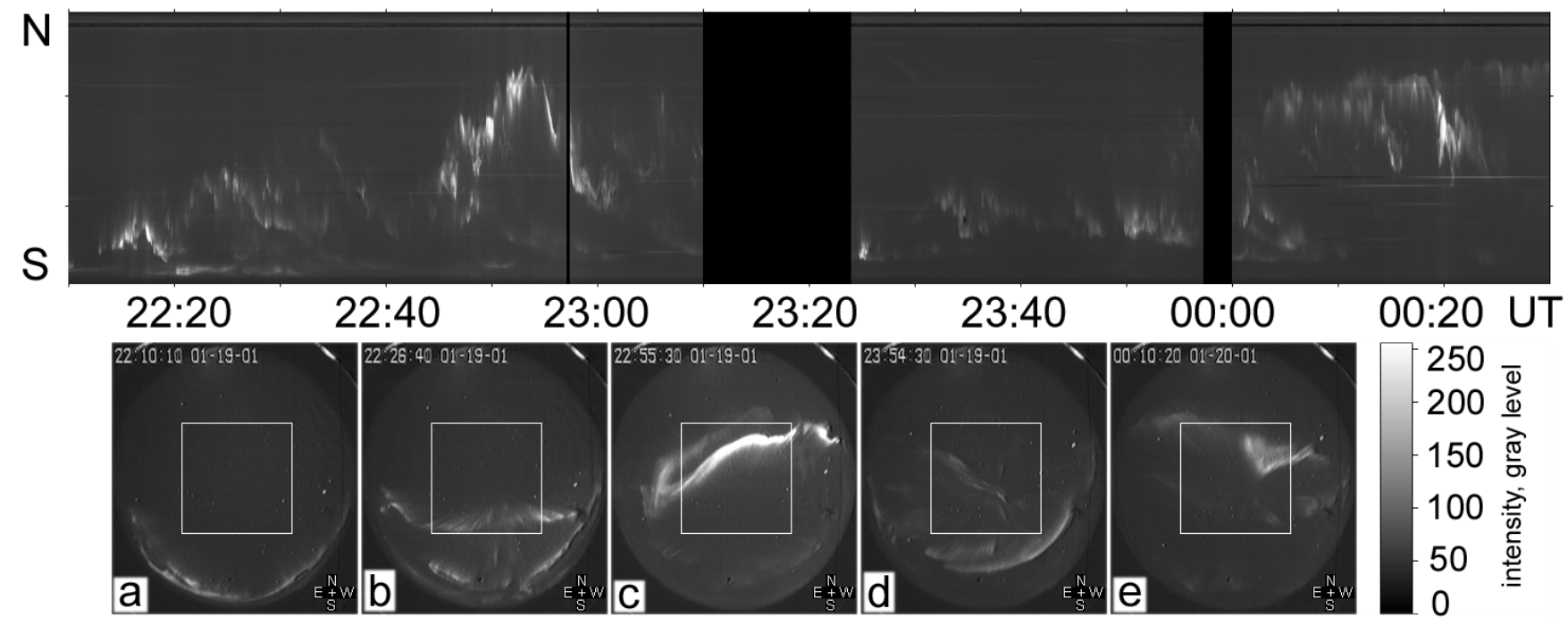

Fig. 1. Auroral structures during the event of 19-20 January 2001. Top panel: north-south keogram obtained from TV ASC images. Bottom panel: several examples of the TV ASC images. The considered part of the field of view is marked by ticks at the vertical axes of the keogram and by white frame in the images.

how the PDFs on different scales can collapse to a single curve under a simple scaling transformation. The GSFs of higher (up to 6) order moments is analyzed in Sect. 4. The evolution of the scaling indices associated with the GSFs of the first three moments during the substorm event is also presented. The main results are discussed and some conclusions are drawn in Sect. 5.

\section{TV observations of aurora}

The Barentsburg observatory is located at Svalbard $\left(78.1^{\circ} \mathrm{N}\right.$, $\left.14.24^{\circ} \mathrm{E}, 75.17^{\circ} \mathrm{MLAT}, 112.1^{\circ} \mathrm{MLON}\right)$, the quiet nighttime auroral oval being observed near its southern horizon. This location is very favorable for observations of poleward motion of aurora during the expansion phase of a substorm. We choose for the analysis the same event during the evening 1920 January 2001, which was previously analyzed in Kozelov et al. (2004), by application of spatio-temporal techniques to identify avalanche-like transients.

The digitized video frames (5 frames per second) from a television all-sky camera (TV ASC) are superposed and averaged over $1 \mathrm{~s}$ to reduce the noise level. The central part of the frames, $\sim 200 \times 200 \mathrm{~km}^{2}$, where the projection distortions are not significant, is used for the analysis. From the geometry of observation, the spatial resolution of the TV ASC in the center of the field of view for the altitude of the aurora $(\sim 110 \mathrm{~km})$ is $\sim 1.2 \mathrm{~km}$, increasing to $\sim 1.7 \mathrm{~km}$ toward the boundary of the area used for analysis. In the analysis we neglect these variations in spatial resolution and other distortions of the ASC image and assume that the average spatial resolution is $\sim 1.5 \mathrm{~km}$. An intensity calibration of TV ASC for this event has been provided in Kozelov (2005) by employing data of simultaneous observations by meridian scanning photometers. In the chosen central field of view the level $\sim 50$ corresponds to the black level and one step of the gray level corresponds to $\sim 60 \mathrm{R}$ of auroral intensity in the line $557.7 \mathrm{~nm}$, or to $\sim 5 \times 10^{-5} \mathrm{Joule} / \mathrm{m}^{2} \mathrm{~s}$ of precipitated particle flux.

Figure 1 presents a keogram (temporal dependence of observed intensity in the north-south section of the TV ASC images) for the substorm event and several TV images from the set. The central part of the frames used in this study is marked by a white rectangle. The Universal Time (UT) and the date of observation are shown in the top of each image. The images illustrate the various types of auroral structures observed during the event. The image " $a$ " is an example with no aurora in the central field of view. The structure in the image "b" is a weak rayed arc; the "c" is a bright arc which tends to develop into a spiral; and the "d" and "e" are coronalike structures. Auroral intensity distributions in short 20-s intervals containing the images shown are chosen as samples for detailed analysis in this paper.

The analysis of the aurora structure from TV data is complicated because of the interference of noise in the registration system, starlight and airglow. Figure 2 shows examples of occurrence distribution of intensity in the central field of view. The distributions have been averaged over 20 successive seconds, therefore values less than level 1 (marked by a dashed line) implies that pixels with such an intensity do not appear in all frames during the period of averaging.

The distribution plotted by a black curve has been obtained for a time interval with no aurora in the field of view (see image "a" in the bottom panel of Fig. 1). The Gaussian core 
of the distributions is concentrated near the value $I \sim 50$, the wings of the core is somewhat wider due to starlight and airglow. Only a few peaks (due to the brightest stars) with an occurrence level less than 1 is observed at higher intensities.

Other distributions presented in Fig. 2 have been obtained for time intervals when auroral structures were located in the central field of view. These distributions have a clearly skewed shape. In the presence of aurora the peak value of the distribution is shifted towards higher intensity. The right tail of the distribution is generally heavier than the left tail, but with the exception of the distribution for the interval starting from 22:55:00 UT, they all fall below the occurrence levels less than 1 pixel within the intensity range registered by TV ASC. This means that the full range of auroral intensity for these structures is well registered by TV ASC. The exception is the interval starting from 22:55:00 UT, corresponding to image "c" in Fig. 1. Here the dynamical range of the TV ASC did not cover the highest auroral intensities.

\section{Probability density functions of auroral fluctuations}

More detailed information may be obtained by analysis of the probability density functions (PDFs) of a two-pixel variation $\delta I$ of the intensity. We consider a two-pixel variation $\delta I(s)=I\left(p_{1}\right)-I\left(p_{2}\right)$ of the intensity $I$, where $p_{1}$ and $p_{2}$ are pixels inside the considered frame and a vertical or horizontal distance between them is equal to $s$. We used $s$ values from 1.5 to $72 \mathrm{~km}$. Each pair of pixels is used twice in the statistics with a different sign of $\delta I$; therefore, the considered PDFs are symmetric by definition. The pairs from 20 successive images have been combined to improve statistics.

Examples of the PDF $P(\delta I, s)$ as a function of intensity variation $\delta I$ for several spatial scales $s$ are shown in Fig. 3. Figure 4 presents the positive tail of the same PDFs in log$\log$ plots. To construct the PDFs of the intensity fluctuations we have used the same time intervals as for the distributions of intensity in Fig. 2. Figure 5 and the insets in Fig. 3 illustrate the scale dependence of the important characteristics of the PDFs: the maximum value $P(0, s)$, the standard deviation $\sigma(s)$ and kurtosis $K(s) . P(0, s)$ is more correctly termed "the probability of the return to the origin", but for these symmetric PDFs it always corresponds to the maximum value of the PDF curve. These parameters have been calculated by utilizing all the available data points without conditioning (see next section). The kurtosis parameter $K(s)$ considered here is defined as

$K(s)=\frac{1}{N} \sum_{p=1 \ldots N}\left(\frac{(\delta I(p, s)-\langle\delta I\rangle)}{\sigma}\right)^{4}-3$,

where $\langle\delta I\rangle$ and $\sigma$ are the mean value and the standard deviation of the intensity fluctuations on a given spatial scale $s$, with the angle brackets imply an averaging over position $p$ in the considered field of view.

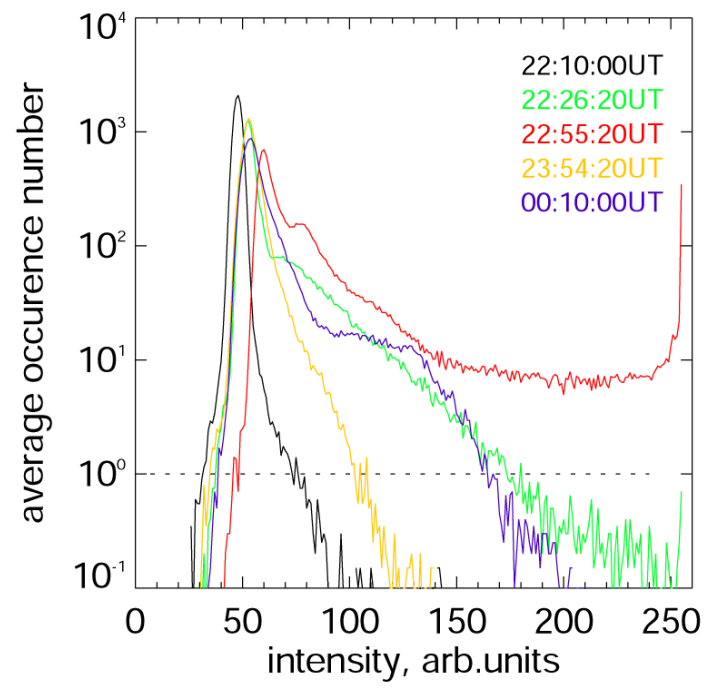

Fig. 2. Occurence number of pixels vs. auroral intensity averaged in 20 -s intervals, beginning from the marked time. 1-pixel occurence level is shown by dashed line.

It is observed that for a time interval without aurora (Fig. 3a) the PDF is practically independent of the scale $s$. There is only a small change in the shape of the central part ("core") of the PDF at $|\delta I|<8$ with an increase in the scale from 3 to $72 \mathrm{~km}$. This leads to some decrease in the PDF maximum $P(0, s)$. However, the standard deviation and kurtosis values are constant because these quantities are mostly determined by the unchanged tails of the distribution. Both tails are symmetrically repeating the shape of the right tail of the occurrence distribution of intensity (black curve in Fig. 2). It is interesting to note that even though the distribution looks like a combination of several Gaussian distributions, the kurtosis parameter has a high value $(K \sim 120)$. The PDF for the smallest scale $s=1.5 \mathrm{~km}$ noticeably differ from the PDFs for the other scales. A possible reason for this is a correlation between values of intensity in neighboring pixels due to the finite bandwidth of TV signal detection, which implies that in the stationary night sky a bright star may occupy more than one pixel. Another possible reason with the same result is a motion of the aurora with a velocity more than $\sim 1.5 \mathrm{~km} / \mathrm{s}$, but this is not the case in the present data.

When the aurora appears in the TV ASC field of view, the PDFs of intensity fluctuations deviate from the simple form characterized by the night sky. These PDFs have a clearly non-Gaussian leptokurtic shape (Figs. 3b-e), and there is a strong dependence on spatial scale. Typically, the PDFs are wider for larger spatial scale, which is seen from the increase in the standard deviation with increasing scale (Figs. 5be). Frequently the tails of the PDFs exhibit a power-law region, which is observed as nearly straight curves in portions of the log-log plots presented in Fig. 4. One can also observe a decrease in the kurtosis parameter with increasing 

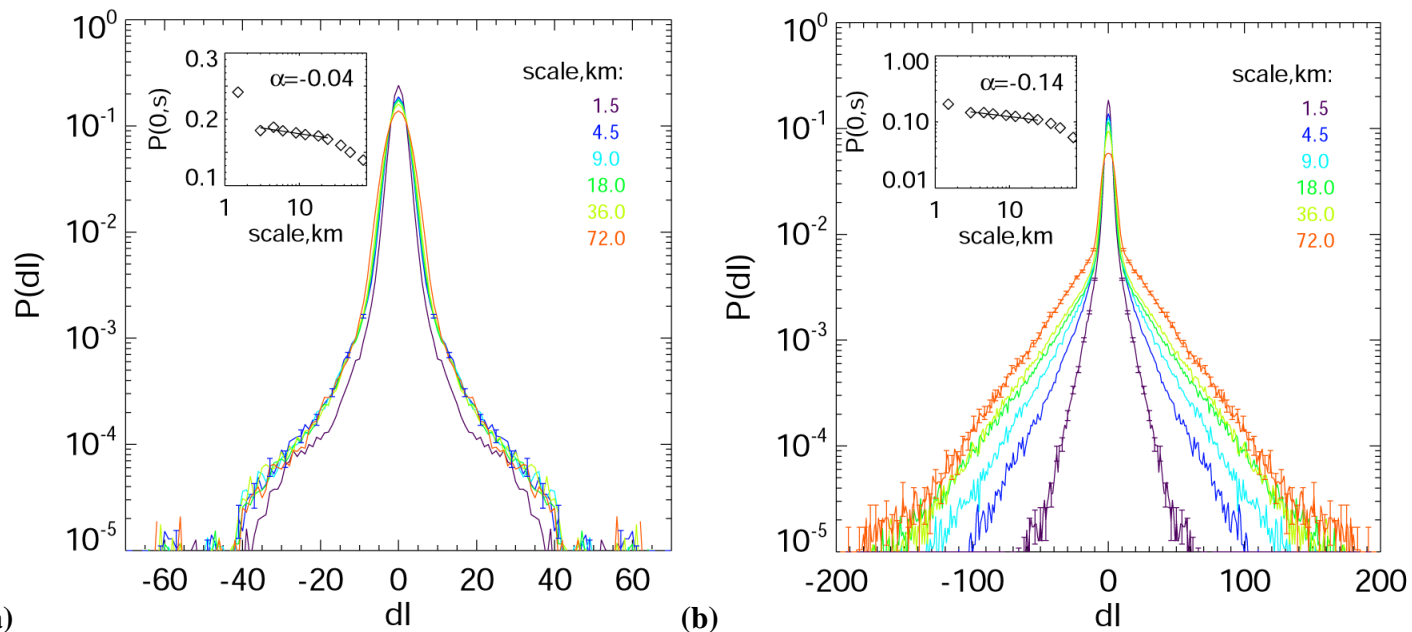

(a)

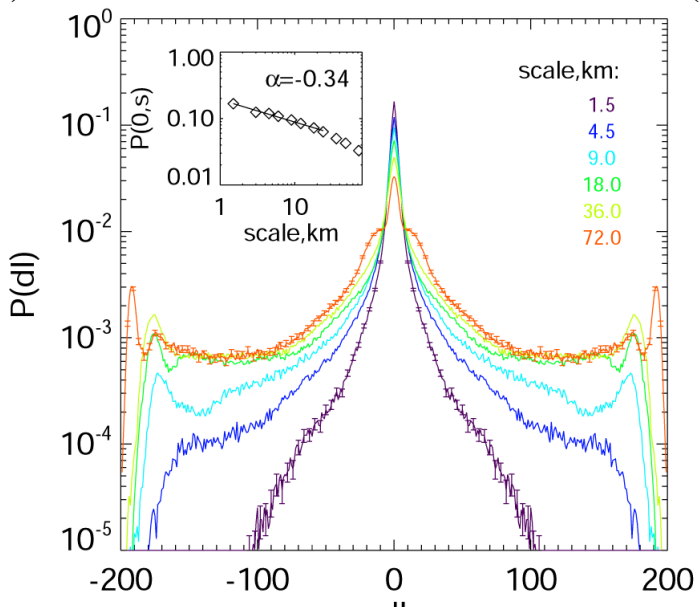

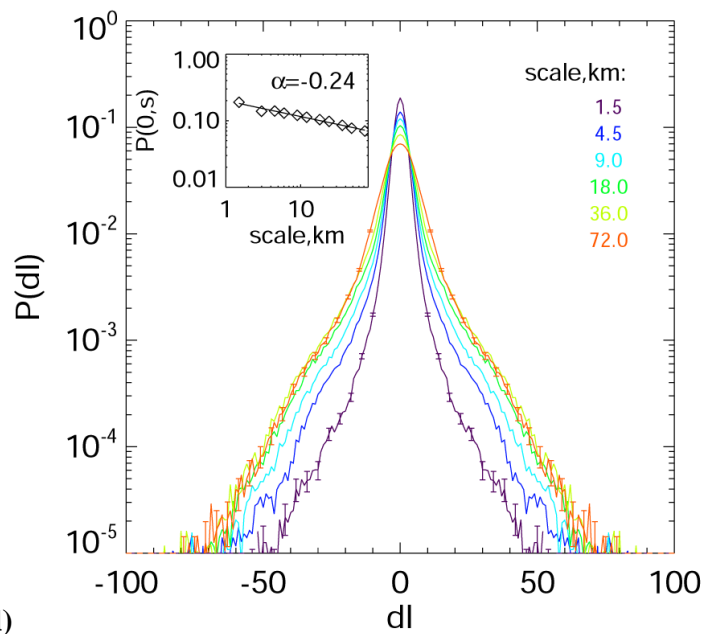

(c)

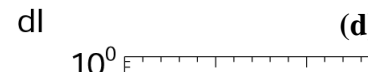

(d)

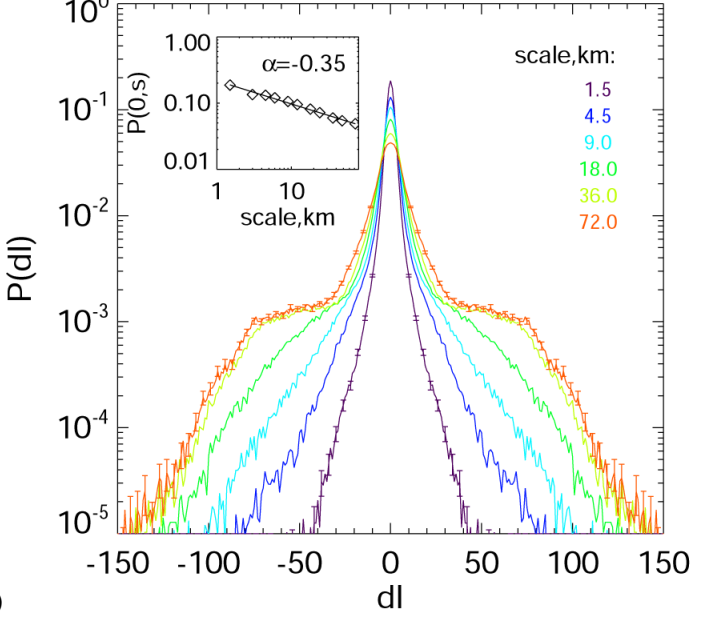

Fig. 3. Dependence of PDF of intensity fluctuations on spatial scale for several $20 \mathrm{~s}$ intervals starting from: (a) 22:10:00; (b) 22:26:20; (c) 22:55:20; (d) 23:54:20; (e) 00:10:00. Insets: symbols - maximum values of the PDF, line - the least sqaures power-law approximation as $\mathrm{P}(0, s) \propto s^{\alpha}$. Uncertainty in the $\alpha$ values is less than 0.01 . 


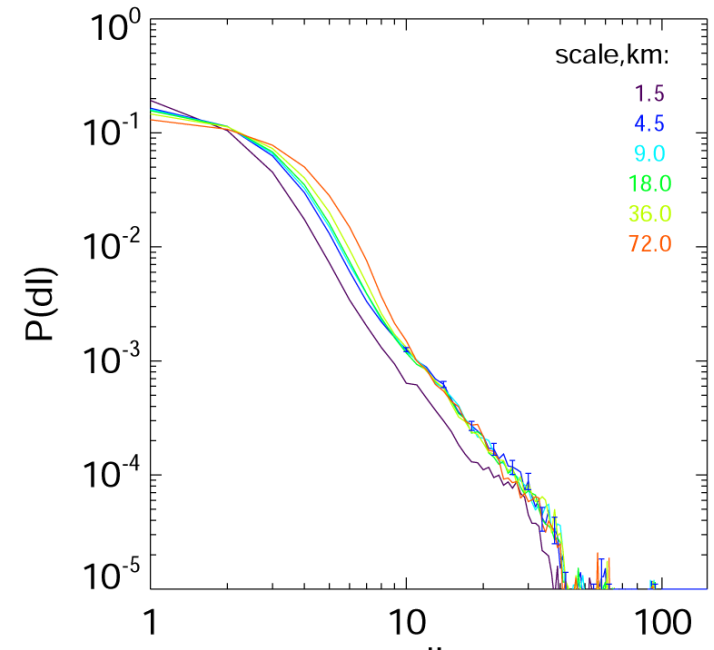

(a)

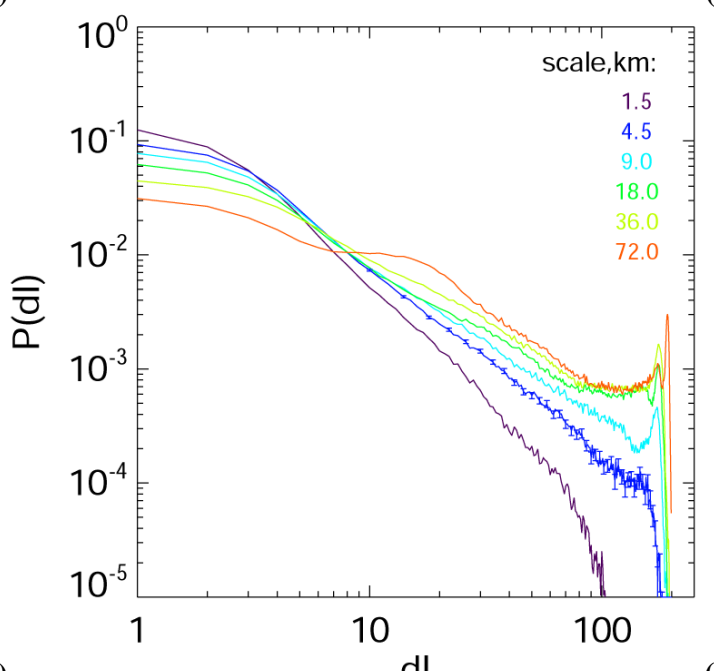

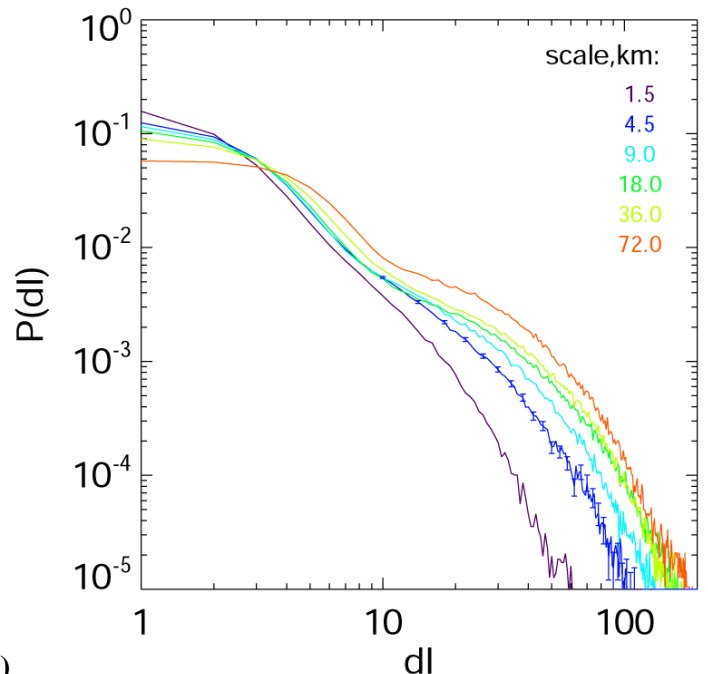

(b)

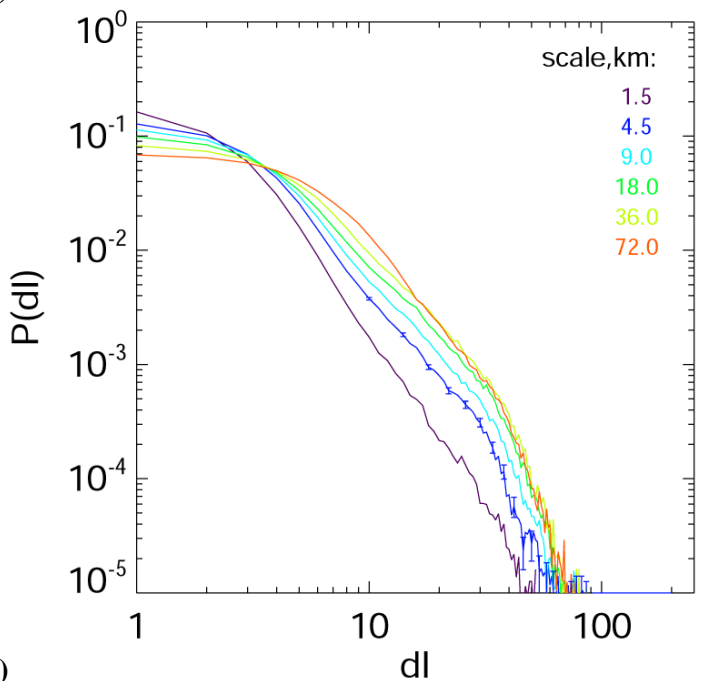

(c)

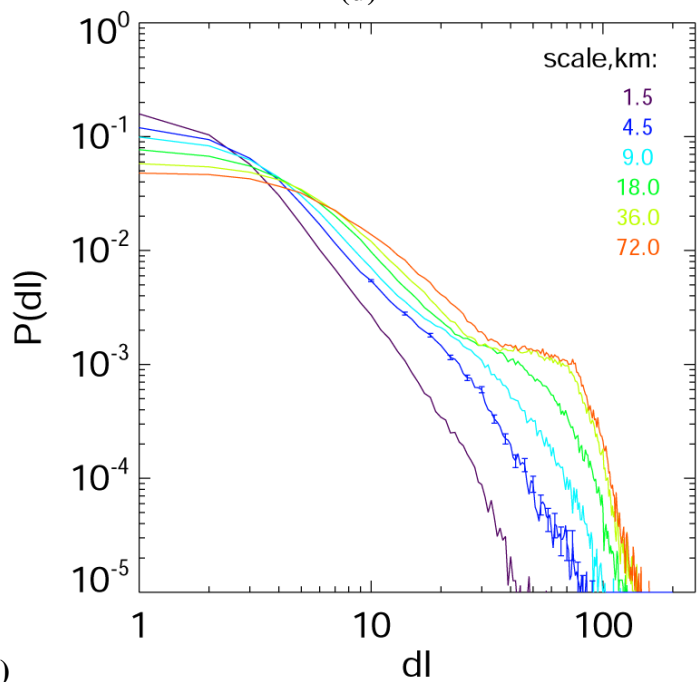

Fig. 4. The same as Fig. 3, but in log-log plots. 


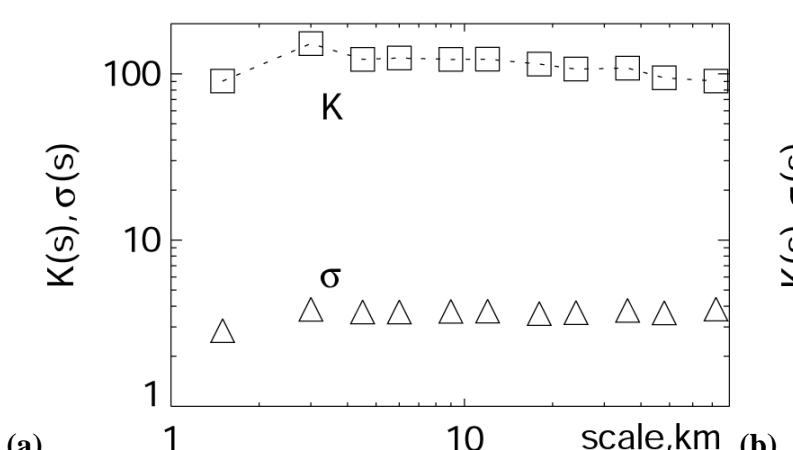

(a)

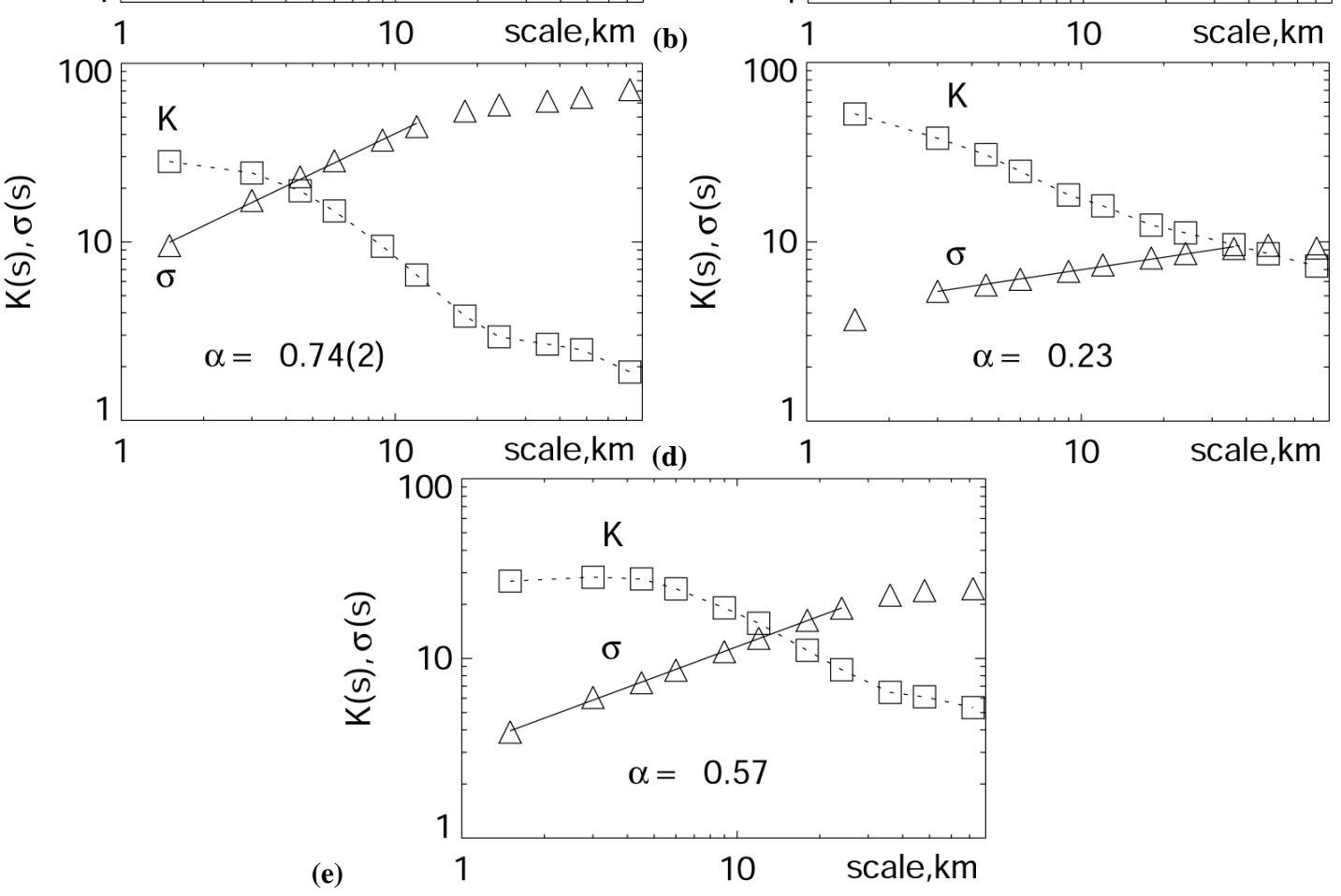

Fig. 5. Scale dependence of the standard deviation $\sigma$ and kurtosis $K$ for PDFs presented in Fig. 3. The region of the least-squares power-law approximation in the $\sigma$ plot is shown by solid line, $\alpha$ is a power index of the approximation. Uncertainty of the $\alpha$ values is less than last decimal unit or they are presented in brackets.

scale (Figs. 5b-e). This is in agreement with the finding of Tam et al. (2005) for electric field fluctuations in the auroral zone. Increasing values of the kurtosis parameter for small scales is normally taken as an indication that the fluctuations are strongly intermittent. It is easily shown (Frisch, 1995) that the kurtosis of a random signal increases with increased burstyness. Intermittency in this sense means that the signal is more bursty on small scales than large scales. However, as will be discussed below, part of this increase in kurtosis may, in some cases, be attributed to a bi-modal nature of the fluctuations, i.e. to the existence of two nearly scale-invariant distributions with different scaling exponents.

The PDFs for the interval 22:55:20-22:56:40 UT (Fig. 3c and Fig. 4c) differ qualitatively from the auroras considered in other time intervals in that they exhibit heavy tails which decay more slowly than $\sim \delta I^{-2}$. As a consequence, the PDFs are wider than the dynamical range of TV observation, and this means that the second moment of the distribution (the standard deviation) for large scales cannot be reliably computed due to the instrumental truncation of the tail. Hence, the values of the standard deviation $\sigma$ and kurtosis $K$ for scales $s>10 \mathrm{~km}$ (Fig. 5c) should be considered only as crude estimates. (The standard deviation is probably underestimated, and the kurtosis overestimated.) For other time intervals, the PDFs are within the instrumental range of detection, thus this limitation poses no problem for estimates of higher moments in those cases.

When an aurora occurs, the scale dependence of the maximum PDF value $P(0, s)$, as well as the standard deviation $\sigma(s)$, exhibit a power-law range, at least for scales from 3 to $20 \mathrm{~km}$. For self-similar PDFs, the power exponents for these two quantities should have the same absolute value, 
and differ in sign, but in these data the absolute value of the power exponent for $P(0, s)$ is typically smaller than for $\sigma(s)$. One exception is the case " $\mathrm{d}$ ", for which the exponents are the same within the accuracy of the estimate. Thus, we conclude that in general the signal under consideration is not self-similar, i.e. it is not a simple fractal characterized by a single scaling exponent.

For a self-similar signal one can collapse the PDFs for a different scale $s$ onto a single curve by scaling the relation $P_{s}\left(d I_{s}\right)=s^{\alpha} P(\delta I, s)$, where $d I_{s}=\delta I / s^{\alpha}$ (Hnat et al., 2003). Attempts of such PDF re-scaling is presented in Fig. 6. For those cases when we have two different values of the scaling exponent $\alpha$ obtained from $P(0, s)$ and $\sigma(s)$, both values were tested.

The PDFs for the case without aurora are perfectly collapsed by the scaling exponent $|\alpha|=0.04$ obtained from the $P(0, s)$ dependence on $s$ (Fig. 6a). Only the PDF for the smallest scale, $s=1.5 \mathrm{~km}$, is somewhat narrower than the collapsed curve. The collapse phenomenon is mainly observed for the core of the distribution, for $\delta I<8$. The tail of the PDFs for $\delta I>8$, for all scales, is practically the same in both re-scaled and non-scaled distributions.

For the case "b" (see Fig. 6b) there are two ranges of the distribution which collapse by different values of $\alpha$ : the core $(\delta I<8)$ - by the value from $P(0, s)$, and the tails - by the value from $\sigma(s)$. The PDFs for the smallest scale, $s=1.5 \mathrm{~km}$, is somewhat narrower than the collapsed curve; hence, we conclude that the collapse of the PDFs tails is observed for scales $s=3-24 \mathrm{~km}$.

There is no scaling collapse of PDFs for case "c" by the scaling exponent obtained from $P(0, s)$, see Fig. 6c. However, for the smaller scales the tails of the PDFs tend to collapse by the scaling exponents for $\sigma(s)$.

For the case "d" the scaling exponents for $P(0, \mathrm{~s})$ and $\sigma(s)$ are the same. One can see from Fig. 6d that the PDFs for scales $s=3-24 \mathrm{~km}$ are reasonably well collapsed, both near the maximum and in the tail region. In the central part of the distribution $\left(d I_{s}=6-20\right)$ the discrepancy of the rescaled curves is somewhat higher ( $\sim$ factor 2 ).

The scaling features of the case "e" are practically the same as for case "b". But the collapse of the PDFs tails is observed for a shorter range of scales, $s=3-12 \mathrm{~km}$.

The dual nature of the scaling collapse (different exponents for core and tail) observed in case "b" and "e" suggests a bifractal structure of the analyzed signal. However, the absence of scaling collapse observed in case "c", combined with the increased kurtosis for small scales, indicates a strong multifractal intermittent structure for this case of a bright auroral arc. The bifractal structure is absent in case "d", and even though the renormalized PDFs collapse reasonably well with one scaling exponent, the increased kurtosis on smaller scales suggests weak multifractal intermittency for this case of a corona-like auroral structure. Multifractality may be investigated by Generalized Structure Functions (GSF), which we discuss in the next section.

\section{Scaling features of generalized structure functions}

The spatial generalized structure function (GSF) at a given time is defined for our problem as

$S_{m}(s)=\left\langle|\delta I|^{m}\right\rangle=\int_{-\infty}^{\infty}|\delta I|^{m} P(\delta I, s) d(\delta I)$,

where $\delta I(p s)=I(p+s)-I(p)$, and the angle brackets imply averaging over position $p$. For PDFs with heavy tails one faces the problem that the theoretical higher order structure functions may be very large or even infinite, and hence that the GSFs computed from the PDFs obtained from the observation data are determined by the size of the available data set. A larger set of independent data points yields heavier tails of the observed distribution, and the computed GSF will be larger. The number of relatively independent values of the fluctuation increment $\delta I(p s)$ will decrease with increasing scale $s$. Hence, for large $s$ the tails of the renormalized observational PDF will be more depleted due to a smaller number of independent data points, and this results in a more severe underestimation of the higher order GSFs. For a selfsimilar signal with a finite $m^{\prime}$ th moment, it is easy to show that $S_{m}(s) \propto s^{\zeta(m)}$, where $\zeta(m)=\alpha m$ with $\alpha$ being constant. If the tails are depleted due to poor statistics at large scales $s$, the scaling exponents $\zeta(m)$ are underestimated, and more so for higher orders of $m$. This leads to a weaker than linear growth of $\zeta$ with $m$, which can be incorrectly interpreted as intermittency. To avoid this problem of poor statistical representation of the PDF tails for larger scales, Hnat et al. (2003) suggest a conditioning technique (Kovács et al., 2001). Under conditioning, the GSF for our case can be expressed via the PDF of the fluctuations as:

$S_{m}(s)=\int_{-A}^{A}|\delta I|^{m} P(\delta I, s) d(\delta I)$.

Here the choice of the threshold $A$ is based on the standard deviation of the intensity fluctuations at a given scale $s, A(s)=C \sigma(s)$. In this work we have adopted $C=10$. We also consider non-conditioned GSF, using all available data.

If the GSF (conditioned or non-conditioned) exhibit scaling with respect to spatial scale $s$, then $S_{m}(s) \propto s^{\zeta(m)}$. This appears to be the case for the auroral fluctuations being examined in this paper in the range of 3-20 km. The examples for the cases "b", "d" and "e" discussed in the previous section are presented in Fig. 7. The case "c" is not considered here because of the instrumental truncation of the power-law tails of the fluctuation PDFs.

From the inset plots in the figure (non-conditioned cases are not shown) one observes that the structure functions of the moment order $\mathrm{m}=1, \ldots, 6$ exhibit a well-defined powerlaw form in the range from $3 \mathrm{~km}$ to $20 \mathrm{~km}$, so it is possible to estimate the scaling exponents $\zeta(m)$. However, the power-law exponents $\zeta(m)$ for non-conditioned GSF, being plotted as a function of the moment order as triangles in 

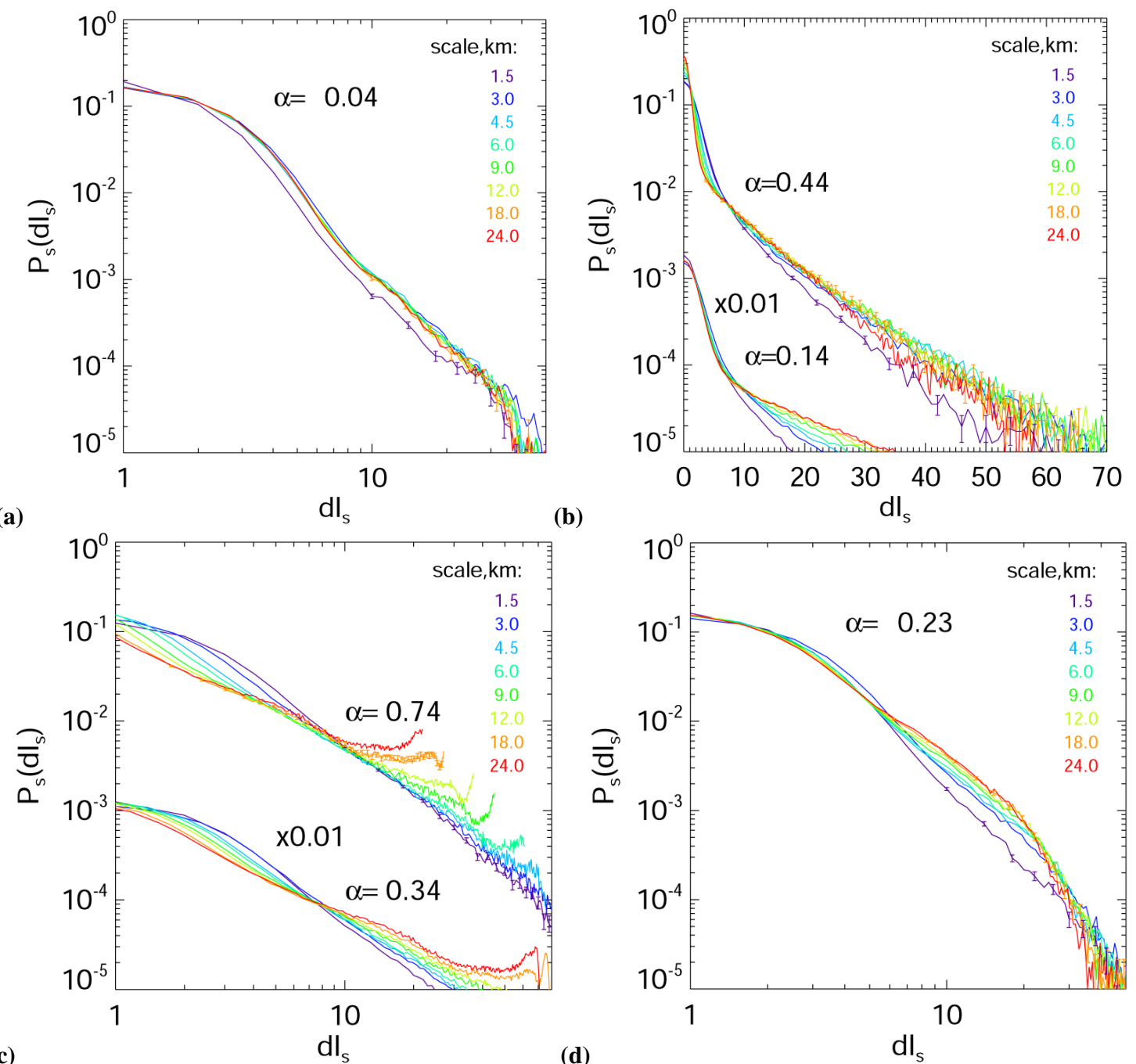

(c)

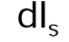

(d)

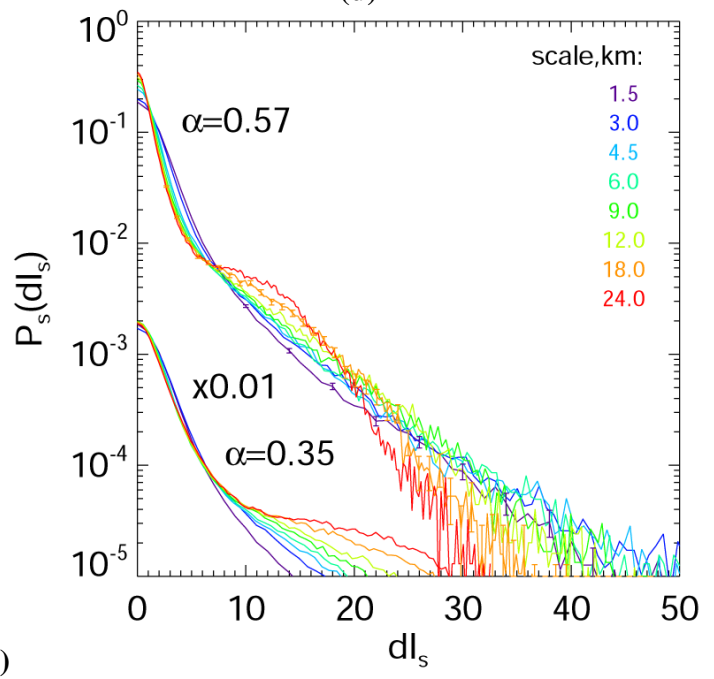

Fig. 6. Scaling collapse of the PDFs for cases from Fig. 3. For (b), (c), and (d) - two sets of curves are presented with different values of the scaling index. 

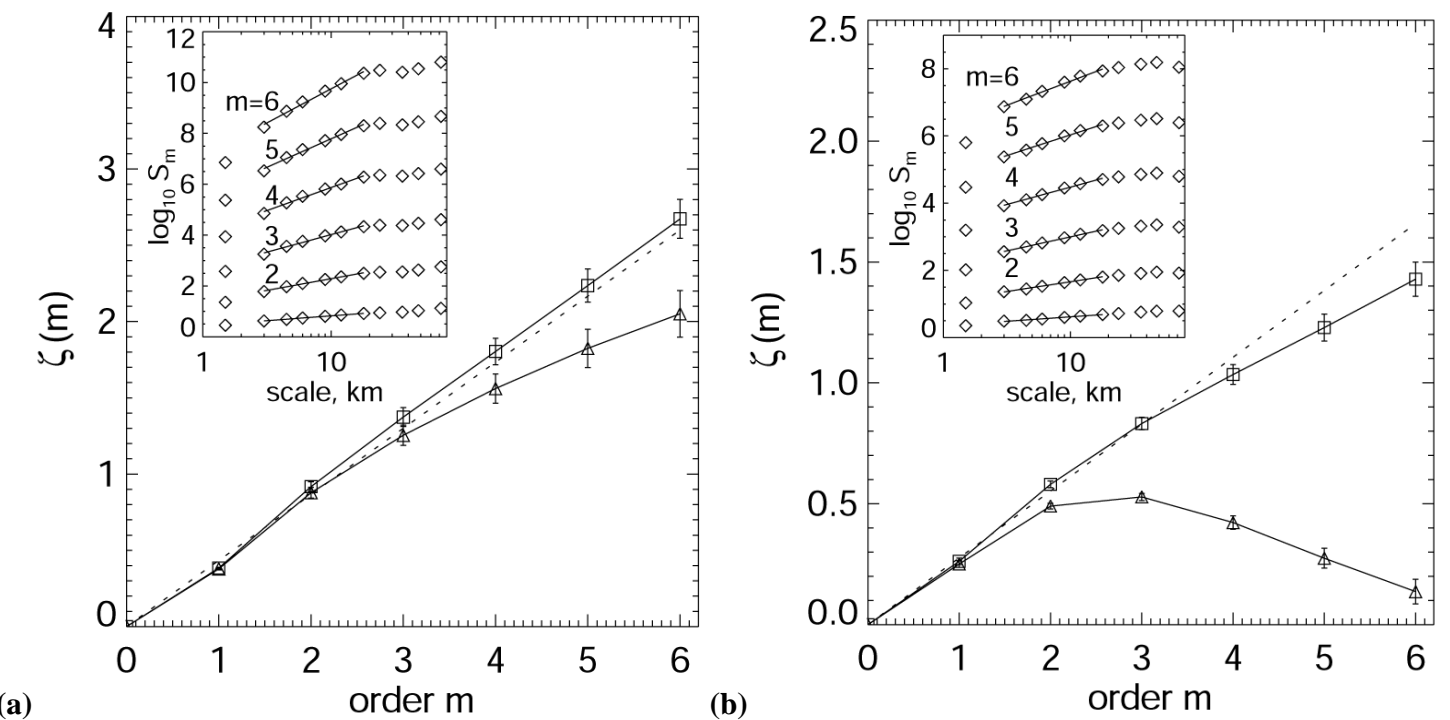

(a)

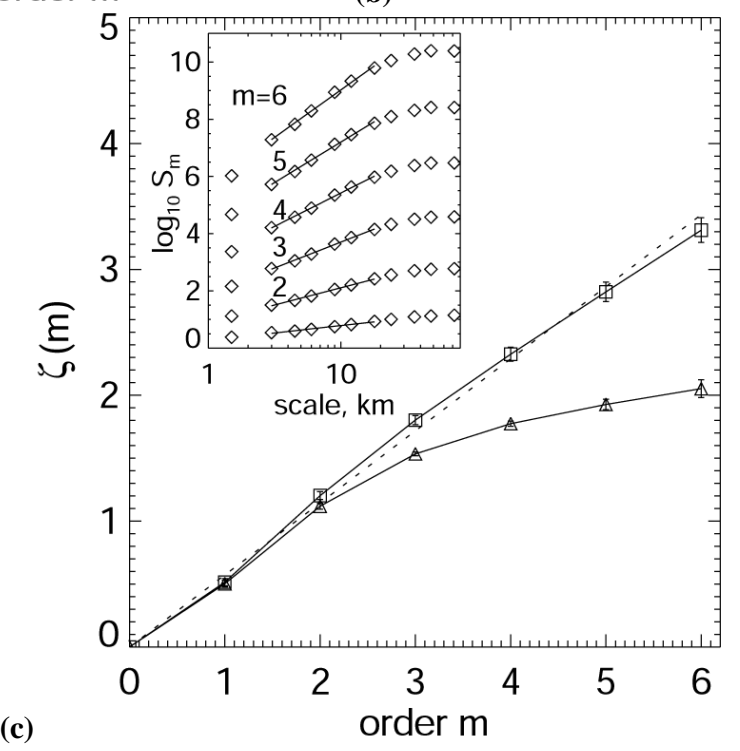

Fig. 7. Exponents of conditioned (squares) and unconditioned (triangles) generalized structure function as a function of the order of $m$ for auroral intensity fluctuation in 20-s interval starting from: (a) 22:26:20; (b) 23:54:20; (c) 00:10:20. Insets: conditioned structure function $S_{m}$ of orders $m=1-6$; solid lines are the least-squares power-law approximation.

Fig. 7, exhibit a pronounced nonlinear dependence on $m$, a feature which is often interpreted as the presence of multifractal intermittency in the signal (Frisch, 1995). However, for the cases of bi-modal distributions the power-law exponents $\zeta(m)$ for conditioned GSF exhibit nearly linear relationships on $m$, as seen in Figs. 7a, c. This suggests that the increased kurtosis for small scales is caused by this bimodality and not by multifractal intermittency, and that the conditioned structure function method is relatively insensitive to this bi-modality. On the other hand, for the case "d", which displays no clear bi-modal PDF, Fig. 7b shows that $\zeta(m)$ deviates from a linear relationship for higher orders $(m>3)$. This deviation should be attributed to true multi- fractal intermittency, although the deviation from monofractal behaviour is not very large.

These nonlinear features of the structure function are observed only when active aurora appears in the considered field of view. Quiet time aurora and weak airglow luminosity does not demonstrate such bi-modal or multifractal features. These categories of events will be considered in detail in forthcoming papers.

Since we have a few hours of observation in the course of the event it is possible to follow the temporal evolution of the scaling exponents. The temporal evolution of the normalized exponents $\zeta(m) / m$ for $m=1,2,3$ is presented in Fig. 8 . The values have been computed by the least-squares power 


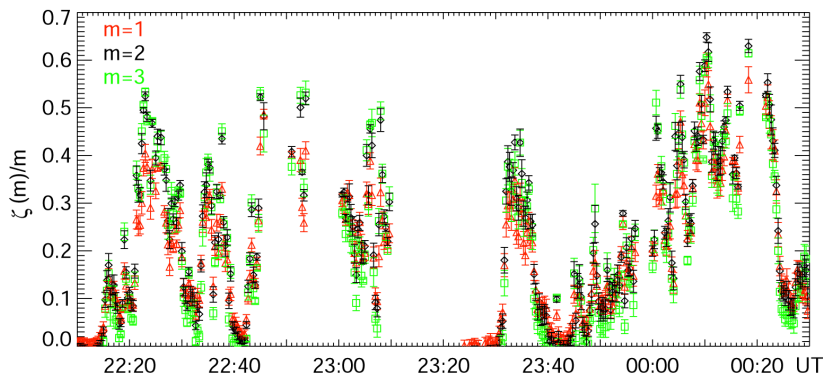

Fig. 8. Evolution of the normalized scaling exponent of conditioned GSF for orders $m=1-3$ during the substorm event.

law approximation of the conditioned GSF in the range of the scales $s=3-20 \mathrm{~km}$ for each 20 -s interval of the data. We exclude from the consideration the intervals for which: i) TV ASC range of gray levels does not cover the intensity range of aurora; ii) the standard deviation of the intensity fluctuation $\sigma(s)>25$ for scales $s<20 \mathrm{~km}$ (because we use the conditioning parameter $A=10 \sigma$ and the total number of the intensity levels is 256). It appears that the normalized exponents $\zeta(m) / m$ for $m=1,2,3$ significantly vary from values $<0.1$ for time period when no aurora appears in the field of view, to $\sim 0.6$ for the most disturbed periods. The value $H=\zeta(2) / 2$ is the Hurst exponent, therefore, the small values may be interpreted as a spatial anti-persistence of the fluctuations, which is characteristic for random noise. However, keeping in mind the results of our PDF analysis, the time intervals where $H>0.5$ may not represent a transition to persistent fluctuations with long-range spatial dependence, but may rather be a symptom of heavy algebraic tails in the fluctuation PDFs during aurora. This question is important to clarify, since leading turbulence theories predict antipersistence (Kolmogorov: $H=1 / 3$, Kraichnan: $H=1 / 4$ ), while SOC models would predict long-range spatial dependence.

We point out that the scaling exponents of the GSFs are invariant to linear transformation of the auroral intensity. Thus, the exponents do not depend (in reasonable limits) on the parameters of TV tape digitizing, and the exponents do not depend on temporal variations of amplification and the black level during TV signal recording, if we avoid the periods of intensity saturation, when the PDFs are truncated. Consequently, no intensity calibration is needed to extract these statistical features. This is one of the important advantages of the method, as the intensity calibration of the TV signal never can become very precise.

\section{Discussion and conclusions}

We have considered the same data set of optical observations which was previously used in Kozelov et al. (2004). In that paper the data set was analyzed by application of a spatiotemporal technique of selection of avalanche-like transients, and it was found that the statistical distributions of the characteristics of these transients is consistent with the existence of SOC-like states in the magnetosphere-ionosphere plasma. In the present paper, using information about spatial distribution of the auroral luminosity, we directly analyze the statistics of its spatial fluctuations: PDF at scales from $\sim 1.5$ to $\sim 80 \mathrm{~km}$ and generalized structure functions up to the 6 th order. We have demonstrated that the same data set shares some intermittency features which are known to be fundamental characteristics of classical turbulence (Frisch, 1995). On the other hand, other features, like long-range spatial dependence, may be present in the data, although this is not firmly established at this point. If such features are confirmed by future observations, they should be taken as support of the SOC hypothesis.

The strong noise of the TV data detection considerably complicates the interpretation of the results. The noise also contains contributions from the airglow and the stars. The noise has the strongest influence on small intensity fluctuations and, therefore, affects mainly the "core" of the PDF $(\delta I<8)$ and the GSFs of the orders of $m<2$. However, the noise fluctuations are practically independent of the spatial scales $s \geq 3 \mathrm{~km}$, hence, the main source of the spatial scaling is the auroral structures.

For the same reason, the PDF tails contain the most essential information about the auroral structures. In the presence of the aurora, the tails exhibit "a heavy", non-Gaussian shape. The tails tend to collapse to a given curve with rescaling of the PDFs by an exponent obtained by using the scale dependence of the standard deviation of the intensity fluctuations at scales $s=3-20 \mathrm{~km}$. The obtained decrease in the PDFs kurtosis parameter with increasing scale is in agreement with the finding of Tam et al. (2005) for electric field fluctuations in the auroral zone. The growing values $(>10)$ of the kurtosis for small scales indicate that the fluctuations of the auroral intensity are intermittent.

We found that the GSFs of the orders of $m=1-6$ vs. spatial scale have a power-law regime at scales $s=3-20 \mathrm{~km}$. The power exponent $\zeta(m)$ for non-conditioned GSF has a nonlinear dependence on the GSF order parameter $m$, which for turbulent flows often is interpreted as a manifestation of intermittent turbulence. For GSF conditioned with $A=10 \sigma$ the dependence is mainly linear, but a nonlinear dependence of $\zeta$ on $m$ is sometimes seen for $m>3$. For the first three orders of GSF the deviation from linearity for $\zeta(m)$ is not large, indicating that the main (non-intermittent) part of the spatial structure is well described by one fractal dimension obtained as $\zeta(m) / m$ for $m=2$ or 3 . We found that this fractal dimension is varying in time during the substorm event. Time variations of the fractal structure have been observed in simulations of Rayleigh-Taylor instabilites (Hasegawa et al., 1996), and variations of fractal dimension of isoline of equal intensity have been found for aurora observations in Kozelov (2003). A general theoretical description of such a transient in turbulent flows is still an open problem. 
An open problem is also the relation between auroral structures and plasma disturbances in the magnetosphere. In spite of the fact that the main energy of the substorm transient is located in the magnetosphere plasma sheet, the source of the small-scale structures is not well understood. There is no doubt that the considered bright auroral structures are located at closed magnetic field lines. However, before the expansion phase of the substorm studied here, a quiet aurora was located south of the field of view, and we can assume that the observed poleward motion of the auroral structure corresponds to a plasma transient in the magnetosphere plasma sheet (Yahnin et al., 2006). On the other hand, the small scales of the structure are possibly affected by the acceleration region at distances closer to the Earth. The spatio-temporal technique (Uritsky et al., 2002; Kozelov et al., 2004) gives the uniform scaling features of avalanchelike transients during the substorm expansion phase from scales of a few square kilometers and a few seconds, up to $10^{6}$ square kilometers and a few hours. The analysis of the PDFs and GSFs presented here shows that the features (power-law range of GSF vs. scale, evidence of the intermittence, leptokurtic shape of the PDFs, and collapse of the rescaled PDFs) of spatial auroral fluctuations on scales of 3$20 \mathrm{~km}$ are the same as for the non-substorm event discussed in Kozelov and Golovchanskaya (2006). This indicates that the scaling relations may represent more universal signatures of spatio-temporal complex dynamics than those pertaining to substorm-like transients in the magnetospheric plasma. Recent work on modeling of the magnetotail current sheet (Klimas et al., 2007) shows signatures of SOC type scaling in the Poynting flux, but evidence of intermittent turbulence in the velocity field. Similar conclusions about the coexistence of SOC scaling and intermittent turbulence are drawn for solar flare activity by Uritsky et al. (2006) from a study of extreme ultraviolet images provided by the SOHO spacecraft. Some evidence for more universal validity of such features relating to complex dynamics in the geosciences were also discussed in Nikora and Goring (2001).

Unfortunately, at present there is no general agreement about how to distinguish SOC and turbulence dynamics from a given set of observation data, in particular for those cases when a velocity field is not directly observable, and we have to base the analysis on a scalar intensity field. Mono-scaling in avalanche size distributions is a basic signature of SOC, and has previously been firmly established for the dataset analyzed in this paper. Intermittency is a signature of turbulence, and has also to some degree been found in the present dataset. Intermittency signatures, as they are defined in turbulence theory are difficult to define for the classical sandpile type of SOC models, due to the limited amplitude-range of the dynamical variables: the "occupation number" at a given site is limited by a threshold. However, time-series for various output characteristics from a SOC-model has recently been analyzed for intermittency by the Troms $\emptyset$ group, since intermittency is basically a property of a signal. Spa- tial intermittency has also been studied in these models by spatio-temporal coarse graining of the dynamical variables. These studies, which will the subject of a forthcoming publication, show that monofractality is not universal for all output characteristics of SOC models, and hence it will be difficult to use intermittency as a signature to distinguish turbulence and SOC.

Boffeta et al. (1999) suggested the use of waiting time statistics between bursts as a distinguishing signature, claiming that SOC models yield exponential waiting-time distributions, while turbulence models produce power-law distributed waiting times. They supported this claim by obtaining exponential waiting times from a simulation of the BakTang-Wiesenfeld (BTW) sand pile, and algebraic waiting times from a shell model of turbulence. However, Paczuski et al. (2005) have recently pointed out that in experimental or observational data sets avalanches can be identified in a noisy background only by introducing a finite threshold of activity, which necessarily will lead to identification of bursts within the same avalanche that will be interpreted as separate avalanches. By simulation of the BWT-model they demonstrate that this will lead to clustering of bursts which results in a power-law waiting time distribution, even if the avalanches themselves have exponentially distributed waiting times. These bursts in the activity are due to the fact that avalanches consist of spatially separated active regions, which also give rise to correlated bursts of flux out of the sand pile. Analysis of waiting times between bursts in the flux will also show a power-law distribution, as we will demonstrate in a forthcoming paper. Thus, it seems that power-law waiting times obtained from observational data are not sufficient to exclude SOC dynamics.

In fact, there are striking structural similarities between the shell model of turbulence, which is a high-dimensional dynamical system model, coupling neighboring shells in kspace, and the sand-pile models, which couple neighboring sites in configuration space. The distinction becomes even more diffuse when one realizes that some sand-pile models can be reformulated as high-dimensional, discrete-time dynamical systems which are chaotic, but with the largest Lyapunov exponent that vanishes in the thermodynamic limit (Kruglikov and Rypdal, 2006).

So far it seems an open question as to whether it is possible to discriminate SOC and turbulence from statistical signatures alone, and whether there are geospace systems where both phenomenologies coexist. A distinct difference between the two classes of complex behavior is that SOC systems develop scale-invariance only when the system has been slowly driven to a state where all sites are near a local instability threshold. Strong driving generally drives the system away from scale-invariant critical behavior. Turbulent systems develop (imperfect) scale-invariance, even if they are strongly driven far above the threshold for the instability which gives rise to the onset of turbulence, and in contrast to SOC systems, end up in a laminar state in the limit of a vanishing 
drive. Thus, one cannot exclude that it may be impossible to discriminate SOC and turbulence without obtaining sufficient knowledge about the strength of the drive and physical mechanisms producing the signals that are subject to statistical analysis.

The present paper, as well as the works of Uritsky et al. (2006) and Klimas et al. (2007), add another dimension to this problem by demonstrating that signatures of SOC and intermittent turbulence can be observed from the same physical system. Further observational and theoretical work along these lines are needed to verify or falsify the hypothesis that SOC dynamics and intermittent turbulence can be different aspects of the same complex phenomenon.

Acknowledgements. The work was partly supported by the Norwegian Science Council (grant 171076) and by the Presidium of the Russian Academy of Sciences (RAS) through the basic research program "Solar activity and physical processes in the Sun-Earth system" and by the Division Physical Sciences of RAS through the program "Plasma processes in the solar system".

Topical Editor I. A. Daglis thanks A. Klimas and another referee for their help in evaluating this paper.

\section{References}

Angelopoulos, V., Mukai, T., and Kokubun, S.: Evidence for intermittency in Earth's plasma sheet and implications for selforganized criticality, Phys. Plasmas, 6, 4161-4168, 1999.

Bak, P., Tang, C., and Wiesenfeld, K.: Self-organized criticality, Physical Review, A38 (1), 364-374, 1988.

Bak, P.: How nature works, The science of self-organized criticality, Oxford University Press, 1997.

Bofetta, G., Carbone, V., Giuliani, P., Veltri, P., and Vulpiani, A.: Power laws in solar flares: self-organized criticality or turbulence?, Phys. Rev. Lett., 83(22), 4662-4665, 1999.

Borovsky, J. E., Elphic, R. C., Funsten, H. O., and Thomsen, M. F.: The Earth's plasma sheet as a laboratory for flow turbulence in high-b MHD, J. Plasma Physics, 57(1), 1-34, 1997.

Borovsky, J. E. and Funsten, H. O.: MHD turbulence in the Earth's plasma sheet: Dynamics, dissipation and driving, J. Geophys. Res., 108, 1284, doi:10.1029/ 2002JA009625, 2003.

Consolini, G.: Sandpile cellular automata and magnetospheric dynamics, Proc. of Cosmic Physics in the Year 2000, 58, edited by: Aiello et al., SIF, Bologna, Italy, 123-126, 1997.

Chang, T., Tam, S. W. Y., and Wu, C. C.: Complexity induced anisotropic bimodal intermittent turbulence in space plasmas, Phys. Plasma, 11, 1287-1299, 2004.

Frisch, U.: Turbulence: The Legacy of A. N. Kolmogorov, Cambridge University Press, New York, 1995.

Hasegawa, S., Nishihara, K., and Sakagami, H.: Numerical simulation of mixing by Rayleigh-Taylor instability and its fractal structures, Fractals, 4(3), 241-250, 1996.

Hnat, B., Chapman, S. C., Rowlands, G., Watkins, N. W., and Freeman, M. P.: Scaling in long term data sets of geomagnetic indices and solar wind $\varepsilon$ as seen by WIND spacecraft, Geophys. Res. Lett., 30, 2174-2177, 2003.
Jensen, H. J.: Self-organized criticality. Emergent complex behavior in physical and biological systems, Cambridge University Press, Cambridge, 1998.

Klimas, A., Uritsky, V. M., and Paczuski, M.: Self-Organized Criticality and Intermittent Turbulence in an MHD Current Sheet with a Threshold Instability, arXiv:astro-ph/0701486 v2, 20 January 2007.

Kovács, P., Carbone, V., and Vörös, Z.: Wavelet-based filtering of intermittent events from geomagnetic time series, Planet. Space Sci., 49, 1219-1231, 2001.

Kozelov, B. V. and Golovchanskaya, I. V.: Scaling of electric field fluctuations associated with the aurora during northward IMF, Geophys. Res. Lett., 33, L20109, doi:10.1029/2006GL027798, 2006.

Kozelov, B. V., Uritsky, V. M., and Klimas, A. J.: Power law probability distributions of multiscale auroral dynamics from ground-based TV observations, Geophys. Res. Lett., 31, L20804, doi:10.1029/2004GL020962, 2004.

Kozelov, B. V.: Calibration of TV all-sky data by simultaneous observations of scanning photometer, Proceedings of 31st Annual European Meeting on Atmospheric Studies by Optical Methods, Ambleside, 22-28 August 2004, 37-41, 2005.

Kozelov, B. V.: Fractal approach to description of auroral structure, Ann. Geophys., 21, 2011-2023, 2003, http://www.ann-geophys.net/21/2011/2003/.

Kruglikov, B. and Rypdal, M.: Dynamics and entropy in the Zhang model of self-organized criticality, J. Statist. Phys., 122, 9751039, 2006.

Milovanov, A. V., Zelenyi, L. M., and Zimbardo, G.: Fractal structures and power law spectra in the distant Earth's magnetotail, J. Geophys. Res., 101(A9), 19903-19910, 1996.

Nikora, V. I. and Goring, D. G.: Extended self-similarity in geophysical and geological applications, Mathematical Geology, 33(3), 251-271, 2001.

Paczuski, M., Boettcher, S., and Baesi, M.: Interocurrence Times in the Bak-Tang-Wiesenfeld Sandpile Model: A Comparison with the Observed Statistics of solar Flares, Phys. Rev. Lett., 95, 181 102, doi:10.1103/PhysRevLett.95.181102, 2005.

Sharma, A. S.: Nonlinear dynamical studies of global magnetospheric dynamics, in: Nonlinear waves and chaos in space plasmas, edited by: Hada, T. and Matsumoto, H., Tokyo, TERRAPUB, 359-389, 1997.

Sorriso-Valvo, L., Carbone, V., Giuliani, P., Veltri, P., Bruno, R., Antoni, V., and Martines, E.: Intermittency in plasma turbulence, Planet. Space Sci., 49, 1193-1200, 2001.

Takalo, J., Timonen, J., and Koskinen, H.: Correlation dimension and affinity of AE data and bicolored noise, Geophys. Res. Lett., 20(15), 1527-1530, 1993.

Tam, S. W. Y., Chang, T., Kintner, P. M., and Klatt, E.: Intermittency analyses on the SIERRA measurements of the electric filed fluctuations in the auroral zone, Geophys. Res. Lett., 32, L05109, doi:10.1029/2004GL021445, 2005.

Uritsky, V. M. and Pudovkin M. I.: Low frequency $1 / \mathrm{f}-$ like fluctuations of the AE-index as a possible manifestation of selforganized criticality in the magnetosphere, Ann. Geophys., 16, 1580-1588, 1998, http://www.ann-geophys.net/16/1580/1998/.

Uritsky, V. M., Klimas, A. J., Vassiliadis, D., Chua, D., and Parks, G.: Scale-free statistics of spatiotemporal auroral emis- 
sions as depicted by POLAR UVI images: Dynamic magnetosphere is an avalanching system, J. Geophys. Res., 107(A12), 1426, doi:10.1029/2001JA000281, 2002.

Uritsky, V. M., Paczuski, M., Davila, J. M., and Jones, S. I.: Coexistence of Self-Organized Criticality and Intermittent Turbulence in the Solar corona, arXiv:astro-ph/0610130 v1, 4 October 2006.

Vörös, Z.: Synergetic approach to substorm phenomenon, in: Magnetospheric substorms, edited by Kan, J. R., Geophys. Monograph, 64, 461-467, 1991.
Vörös, Z., Baumjohann, W., Nakamura, R., Runov, A., Zhang, T. L., Volwerk, M., Eichelberger, H. U., Balogh, A., Horbury, T. S., Glaßmeier, K.-H., Klecker, B., and Rème, H.: Multi-scale magnetic field intermittence in the plasma sheet, Ann. Geophys., 21, 1955-1964, 2003, http://www.ann-geophys.net/21/1955/2003/.

Yahnin, A. G., Despirak, I. V., Lubchich, A. A., Kozelov, B. V., Dmitrieva, N. P., Shukhtina, M. A., and Biernat, H. K.: Relationship between substorm auroras and processes in the near-earth magnetotail, Space Sci. Rev., 122, 97-106, doi:10.1007/s11214006-5884-4, 2006. 